\title{
Convalescent Plasma Therapy for COVID-19: A Graphical Mosaic of the Worldwide Evidence
}

\section{OPEN ACCESS}

Edited by:

Yoav Keynan,

University of Manitoba, Canada

Reviewed by:

Charles J. Vukotich Jr.

University of Pittsburgh, United States

Tingtao Chen,

Nanchang University, China

*Correspondence:

Michael J. Joyner

Joyner.Michael@mayo.edu

tThese authors have contributed equally to this work

Specialty section:

This article was submitted to Infectious Diseases - Surveillance,

Prevention and Treatment,

a section of the journal

Frontiers in Medicine

Received: 22 March 2021 Accepted: 04 May 2021

Published: 07 June 2021

Citation:

Klassen SA, Senefeld JW, Senese KA, Johnson PW, Wiggins CC, Baker SE, van Helmond $N$, Bruno $K A$, Pirofski L-a, Shoham S, Grossman BJ, Henderson JP, Wright RS, Fairweather D, Paneth NS,

Carter RE, Casadevall $A$ and Joyner MJ (2021) Convalescent Plasma Therapy for COVID-19: A Graphical Mosaic of the Worldwide Evidence. Front. Med. 8:684151. doi: 10.3389/fmed.2021.684151

\begin{abstract}
Stephen A. Klassen ${ }^{1 \dagger}$, Jonathon W. Senefeld ${ }^{1+}$, Katherine A. Senese ${ }^{1 \dagger}$, Patrick W. Johnson ${ }^{2}$, Chad C. Wiggins ${ }^{1}$, Sarah E. Baker ${ }^{1}$, Noud van Helmond ${ }^{3}$, Katelyn A. Bruno ${ }^{4}$, Liise-anne Pirofski ${ }^{5}$, Shmuel Shoham ${ }^{6}$, Brenda J. Grossman 7 , Jeffrey P. Henderson ${ }^{8}$, R. Scott Wright ${ }^{9}$, DeLisa Fairweather ${ }^{4}$, Nigel S. Paneth ${ }^{10}$, Rickey E. Carter ${ }^{2}$, Arturo Casadevall ${ }^{11 \dagger}$ and Michael J. Joyner ${ }^{1 *+}$
\end{abstract}

${ }^{1}$ Department of Anesthesiology and Perioperative Medicine, Mayo Clinic, Rochester, MN, United States, ${ }^{2}$ Department of Health Sciences Research, Mayo Clinic, Jacksonville, FL, United States, ${ }^{3}$ Department of Anesthesiology, Cooper Medical School of Rowan University, Cooper University Health Care, Camden, NJ, United States, ${ }^{4}$ Department of Cardiovascular Medicine, Mayo Clinic, Jacksonville, FL, United States, ${ }^{5}$ Division of Infectious Diseases, Department of Medicine, Montefiore Medical Center, Albert Einstein College of Medicine, New York, NY, United States, ${ }^{6}$ Division of Infectious Diseases, Johns Hopkins University School of Medicine, Baltimore, MD, United States, ${ }^{7}$ Department of Pathology and Immunology, Washington University School of Medicine in St. Louis, St. Louis, MO, United States, ${ }^{8}$ Division of Infectious Diseases, Department of Medicine, Department of Molecular Microbiology, Washington University School of Medicine in St. Louis, St. Louis, MO, United States, ${ }^{9}$ Department of Cardiovascular Medicine, Human Research Protection Program, Mayo Clinic, Rochester, MN, United States, ${ }^{10}$ Department of Epidemiology and Biostatistics, Department of Pediatrics and Human Development, College of Human Medicine, Michigan State University, East Lansing, MI, United States, ${ }^{11}$ Division of Infectious Diseases, Johns Hopkins University School of Medicine, Baltimore, MD, United States

Convalescent plasma has been used worldwide to treat patients hospitalized with coronavirus disease 2019 (COVID-19) and prevent disease progression. Despite global usage, uncertainty remains regarding plasma efficacy, as randomized controlled trials (RCTs) have provided divergent evidence regarding the survival benefit of convalescent plasma. Here, we argue that during a global health emergency, the mosaic of evidence originating from multiple levels of the epistemic hierarchy should inform contemporary policy and healthcare decisions. Indeed, worldwide matched-control studies have generally found convalescent plasma to improve COVID-19 patient survival, and RCTs have demonstrated a survival benefit when transfused early in the disease course but limited or no benefit later in the disease course when patients required greater supportive therapies. RCTs have also revealed that convalescent plasma transfusion contributes to improved symptomatology and viral clearance. To further investigate the effect of convalescent plasma on patient mortality, we performed a meta-analytical approach to pool daily survival data from all controlled studies that reported Kaplan-Meier survival plots. Qualitative inspection of all available Kaplan-Meier survival data and an aggregate Kaplan-Meier survival plot revealed a directionally consistent pattern among studies arising from multiple levels of the epistemic hierarchy, whereby convalescent plasma transfusion was generally associated with greater patient survival. Given that convalescent plasma has a similar safety profile as standard plasma, convalescent plasma should be implemented within weeks of the onset of future infectious disease outbreaks.

Keywords: convalescent plasma therapy, COVID-19, SARS-CoV-2, passive antibody transfer, Kaplan-Meier analysis 


\section{INTRODUCTION}

In response to the coronavirus disease 2019 (COVID-19) pandemic caused by the novel human severe acute respiratory syndrome coronavirus 2 (SARS-CoV-2), convalescent plasma has been used worldwide to treat hospitalized patients and prevent progression of disease in non-hospitalized patients (14). Due to the limited number of therapeutic alternatives for COVID-19, widespread optimism for antibody therapy has also led to the commercial production of other immunoglobulin therapies, including monoclonal antibodies and hyperimmune products $(5,6)$. However, despite global usage, clinicians and researchers have struggled to reach consensus regarding the clinical utility of convalescent plasma. Although signatures of efficacy have emerged consistently from worldwide matchedcontrol studies (7), there remains a paucity of data from large randomized clinical trials (RCTs) demonstrating efficacy. Recent reviews and meta-analyses have also generated disharmonious conclusions regarding the effect of convalescent plasma on COVID-19 patient outcomes, such as mortality and clinical improvement. A pragmatic meta-analysis by our group that included data from both RCTs and matched-control studies demonstrated a mortality reduction attributed to convalescent plasma in COVID-19 patients (8). Conversely, recent reviews that adhered to stricter study inclusion rules and aggregated only RCT data concluded that either convalescent plasma had no effect on mortality or that insufficient evidence exists to determine efficacy $(9,10)$. In addition, data emerging from the RECOVERY Trial in the United Kingdom showed no survival benefit of convalescent plasma in its overall cohort that was severely ill but demonstrated trends toward efficacy when plasma was transfused to patients who were earlier in the disease course and did not require supplemental oxygen or corticosteroids (11). These contradictory findings present a challenge for physicians in deciding the therapeutic strategy for COVID-19 patients.

We argue that during a global health emergency like a pandemic, evidence originating from multiple levels of the epistemic evidence hierarchy should be used to inform contemporary policy and healthcare decisions. Such an approach is needed for a complex therapy like convalescent plasma where efficacy depends on the quality of the plasma, the timing of administration, and the immunological status of the patient (12). Thus, this graphical review aims to showcase the mosaic of worldwide evidence related to convalescent plasma therapy for COVID-19.

\section{PRINCIPLES OF CONVALESCENT PLASMA THERAPY}

Briefly, convalescent plasma represents a form of passive antibody therapy that relies on the transfer of pathogenspecific antibodies from a recovered patient for the purpose of preventing or treating disease (13). Unlike vaccines and monoclonal antibodies, convalescent plasma therapy requires limited development or infrastructure, requiring no more than the availability of disease survivors willing to donate plasma and standard blood collection infrastructure to collect and distribute convalescent plasma (14). With no vaccines or monoclonal antibodies available at the onset of the COVID-19 pandemic, convalescent plasma was an immediately deployable option $(14,15)$. In addition, for this reason, convalescent plasma can be readily used in low resource settings across the globe.

Convalescent plasma is also adaptable to changing conditions. As variant SARS-CoV-2 strains continue to emerge, convalescent plasma donated by survivors of infections with variant strains represents an immediately deployable therapeutic for patients identified with a variant infection, whereas other immune therapies may require (re)development to more specifically target new viral strains (16-19).

To effectively neutralize SARS-CoV-2 and confer clinical benefit, convalescent plasma must adhere to the three fundamental principles of passive antibody therapy (12). Convalescent plasma must (20-22):

i) Contain specific antibodies against the pathogen, the SARS-CoV-2 virus

ii) Contain a sufficient level of anti-SARS-CoV-2 antibody, and

iii) Be transfused prophylactically or early in the disease course.

\section{CONVALESCENT PLASMA FOR PREVIOUS RESPIRATORY VIRAL OUTBREAKS}

The widespread use of convalescent plasma in the COVID-19 pandemic was founded on its rich history of efficacy against human respiratory viral infections. Indeed, the first Nobel Prize in Physiology or Medicine was awarded for the discovery of convalescent plasma (serum) therapy for diphtheria (15). Since the late nineteenth century, convalescent plasma has been used to mitigate several outbreaks caused by human respiratory viruses. A meta-analysis of eight studies $(n=$ 1,703 patients) found that convalescent plasma reduced the absolute risk of death by $21 \%$ in patients with $\mathrm{H} 1 \mathrm{~N} 1$ viral infections during the 1918 influenza pandemic (23). Subgroup analysis of patients transfused with convalescent plasma within 3 days of illness onset demonstrated a $41 \%$ lower risk of death compared to patients transfused four or more days after illness onset, highlighting an important role for timely convalescent plasma transfusion (23). Convalescent plasma has also been associated with positive clinical outcomes in recent outbreaks caused by other coronaviruses, including the 2001 SARS1 epidemic and the 2012 Middle East Respiratory Syndrome (MERS) (24-26). For instance, in 80 patients diagnosed with SARS1, patients transfused with convalescent plasma within 2 weeks of illness onset were more likely to be discharged by day 22 of hospitalization than patients treated later in the disease course (24). Although most of the historical evidence for convalescent plasma emerged from retrospective matchedcontrol designs and single-arm studies, the favorable efficacy data and positive safety signals provided strong precedent for rapid implementation at the onset of the COVID-19 pandemic $(14,20)$. 


\section{CONVALESCENT PLASMA THERAPY FOR COVID-19: EXPERIMENTAL EVIDENCE}

In the context of the COVID-19 pandemic, the anticipated primary mechanism for the clinical benefit of convalescent plasma immunotherapy is SARS-CoV-2 viral neutralization $(27,28)$. Virus neutralization occurs when antibodies bind to the spike protein and prevent binding to the host cellular receptors. In addition to viral neutralization, convalescent plasma includes antibodies that mediate three other antiviral functions against SARS-CoV-2: (i) complement activation, (ii) antibodydependent cellular cytotoxicity, and (iii) phagocytosis (29). The antiviral effect of convalescent plasma is supported by RCTs and observational studies, which have consistently shown a reduction in viral load following transfusion $(3,27,30)$.

COVID-19 pathogenesis begins with an early viral phase that can progress to a life-threatening inflammatory phase (31). The viral phase is characterized by SARS-CoV-2 virus replication that is accompanied by variable symptoms and triggers an endogenous antibody response around days 10-12 of infection (32). Some individuals may progress to an inflammatory phase that may clear the virus but impairs pulmonary gas exchange and in some cases causes respiratory failure and death $(31,33,34)$. Thus, early convalescent plasma transfusion during the viral phase is effective because viral neutralization prevents disease progression to the severe inflammatory phase. Consistent with this view, convalescent plasma administration in COVID-19 is followed by reduction in markers of inflammation $(35,36)$. A synthesis of these observations suggests that early administration of convalescent plasma reduces viral burden through antibodymediated antiviral effects, which in turn prevents inflammation promoting a survival benefit by improving oxygen exchange in the lung.

\section{Animal Studies}

During the COVID-19 pandemic, several animal studies performed in parallel with human clinical investigations have demonstrated convalescent plasma efficacy in experimental SARS-CoV-2 infection. For example, convalescent serum from Syrian hamsters elicited an antiviral effect when administered to infected animals (37). Similarly, among SARS-CoV-2-infected green monkeys, transfusion of convalescent plasma from the same species reduced lung pathology, viral shedding, and inflammation (38). In addition, administration of human convalescent plasma protected mice expressing the human ACE2 receptor from SARS-CoV-2 infection (39). These studies illustrate that animal and human convalescent plasma contains virus-neutralizing antibodies that mediate protective effects in animal models of SARS-CoV-2 infection.

\section{CONVALESCENT PLASMA THERAPY FOR COVID-19: MOSAIC OF CLINICAL EVIDENCE}

To date, 11 RCTs $(11,27,30,40-47)$ and 24 matched-control studies $(3,4,48-69)$ have investigated convalescent plasma therapy for COVID-19. Of these studies, 16 presented survival data using a Kaplan-Meier diagram (11, 27, 41, 43, 46, 47, 50, $52,59-62,64,69-71)$. To investigate the impact of convalescent plasma on COVID-19 patient survival over time, we extracted daily survival data from all available Kaplan-Meier diagrams using an online data extraction tool (WebPlotDigitizer v4.4, Pacifica, CA, USA). Kaplan-Meier survival plots were replotted for each study. Aggregate Kaplan-Meier survival plots were computed by pooling the derived number of deaths per day from the daily survival rate and reported number of patients at risk. For aggregate survival plots, 95\% confidence intervals were calculated by Greenwood's formula (72). This meta-analytical approach provides greater temporal resolution of survival data compared to conventional meta-analyses that rely on the overall survival rate at the end of the study period (73). Figures were generated with $\mathrm{R}$ software (R Core Team, Vienna, Austria) and GraphPad Prism (GraphPad Software, San Diego, CA, USA).

Qualitative inspection of the available Kaplan-Meier survival data from 16 controlled studies revealed a directionally consistent pattern whereby convalescent plasma transfusion was associated with greater patient survival compared to non-transfused patients (Figure 1) (11, 27, 41, 43, 46, 47, 50, 52, 59-62, 64, 69-71). Pooling all available Kaplan-Meier survival data showed a $14 \%$ relative improvement in COVID-19 patient 28 -day survival associated with convalescent plasma (84 vs. $74 \%$ ) (Figure 2A). We offer the caveat that due to the large sample size of the RECOVERY Trial (11), these data were not included in this aggregation but reported separately (Figure 2D). The RECOVERY Trial found no difference in 28-day mortality between COVID-19 patients treated with convalescent plasma and non-transfused patients (24 vs. $24 \%$ ) (11). The general survival benefit associated with convalescent plasma has been observed worldwide including studies that were heterogenous for health system type and infectious disease or critical care infrastructure, timing relative to pandemic onset, convalescent plasma antibody titer and volume, and patient disease severity (Supplementary Figure 1). This consistency of evidence across nations with different health systems supports the notion that convalescent plasma therapy is effective against COVID-19.

\section{Randomized Clinical Trials}

Signatures of convalescent plasma efficacy have emerged from some but not all RCTs. Inspection of available Kaplan-Meier survival data from seven published RCTs revealed a directionally consistent pattern whereby convalescent plasma appeared to improve COVID-19 patient survival compared to patients that received standard of care (Figure 1) $(11,27,41,43,46,47,71)$. Pooling these available survival data demonstrated a $11 \%$ relative improvement in 28-day survival associated with convalescent plasma compared to control (88 vs. 79\%) (Figure 2B). While the effect of convalescent plasma on mortality was found to be statistically significant in only two RCTs $(42,71)$, it is important to highlight that signals of efficacy have emerged despite several limitations, including trials that (i) were underpowered due to declining local infection rates and early termination $(27,43)$, (ii) transfused convalescent plasma units with unknown or low 


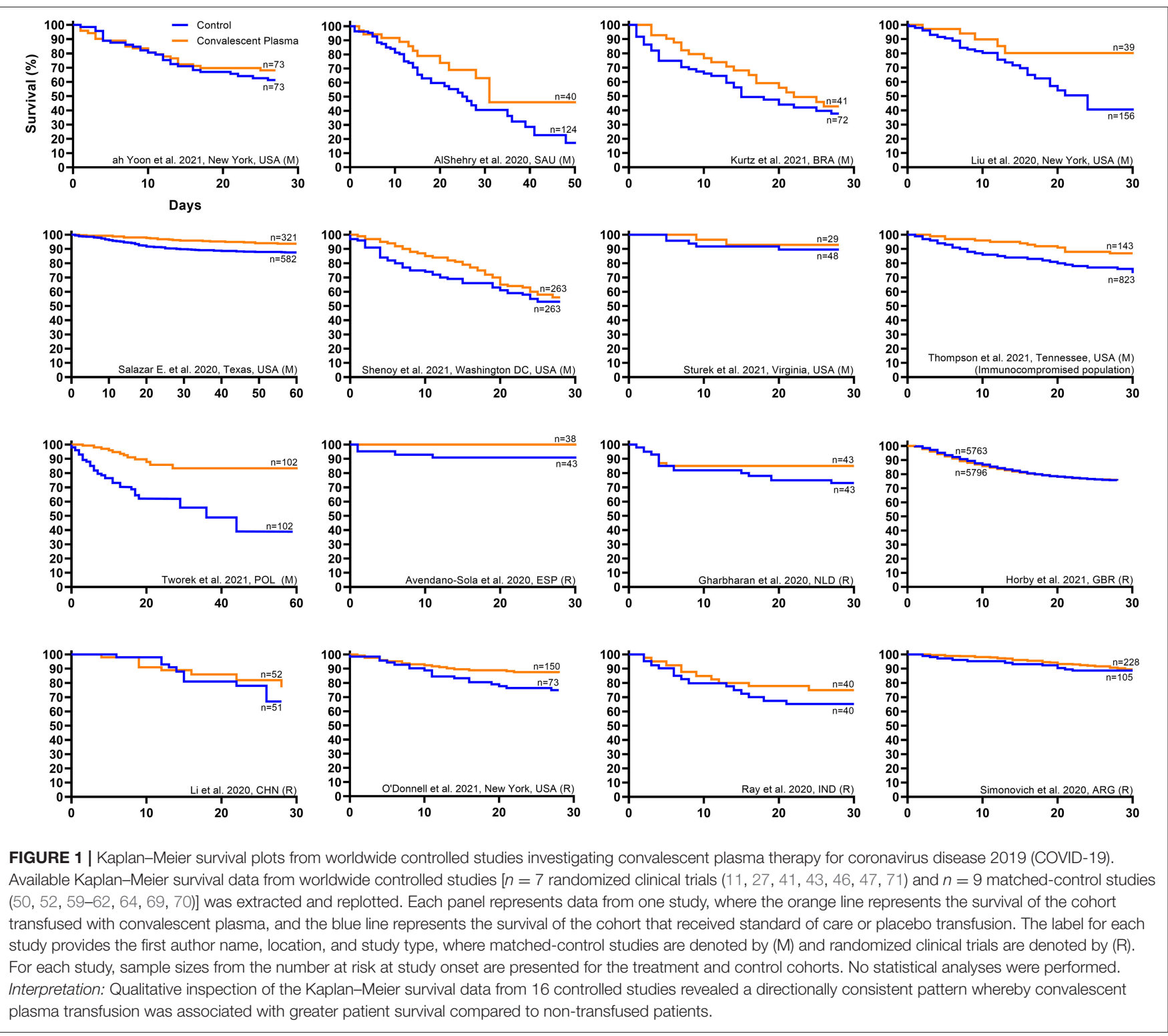

anti-SARS-CoV-2 antibody levels (30), (iii) transfused severely ill patients that had progressed to severe COVID-19 $(11,27)$, and (iv) used prepositioned plasma units, which may not have accounted for SARS-CoV-2 variants $(18,19)$. Transfusion of low antibody titer plasma to patients with severe COVID-19 reduces the opportunity to achieve the survival benefit associated with convalescent plasma. Due to the large sample size of the RECOVERY Trial $[n=11,558$; (11)], these data were not included in the aggregate RCT Kaplan-Meier plot (Figure 2B) but reported separately (Figure 2D). The RECOVERY Trial likely did not find an effect of convalescent plasma in their overall cohort due to the large proportion of severely ill patients ( $24 \%$ overall mortality) who were treated late in the disease course (median time to transfusion, 9 days). Corticosteroids, which interfere with antibody function, were used in more than $90 \%$ of study patients in both arms (11).
Nonetheless, some signals of efficacy emerged from subgroup analyses of the RECOVERY Trial data (11). First, in four likely overlapping groups of patients characterized as representing earlier disease, significant or near-significant lowering of mortality was seen with convalescent plasma: among patients transfused within 7 days of symptom onset [odds ratio, 0.92 (95\% confidence interval, $0.83-1.03)$ ], patients not receiving corticosteroids [odds ratio, 0.78 (95\% confidence interval, $0.58-$ 1.05)], patients not on supplemental oxygen [odds ratio, 0.83 (95\% confidence interval, $0.58-1.18$ )], and patients without an endogenous anti-SARS-CoV-2 antibody response at time of transfusion [see Figure 2E-odds ratio, 0.94 (95\% confidence interval: 0.84-1.06)] (11). These trends toward a survival benefit with early plasma transfusion are consistent with the RCT by Libster et al., which observed a $\sim 50 \%$ reduction in progression to severe COVID-19 respiratory disease when mildly 


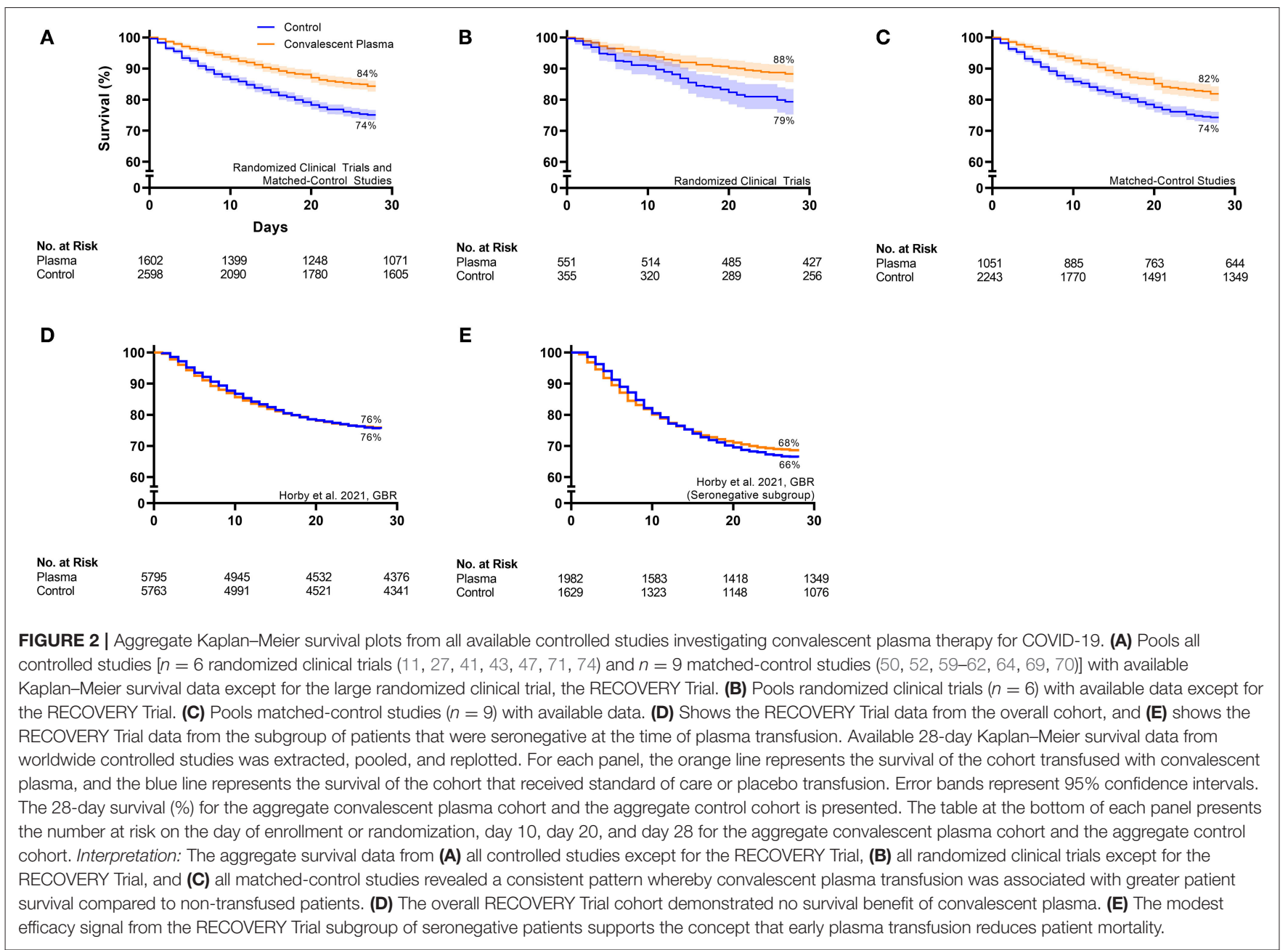

ill patients were transfused with convalescent plasma screened for appropriate level of antibody and a 73\% reduction when plasma with antibody above the median was used (45).

Not all meta-analytical approaches support the impact of convalescent plasma on patient survival. A recent Cochrane review and meta-analysis that was restricted to only a few RCTs found insufficient evidence to draw conclusions regarding plasma efficacy (10). In addition, a meta-analysis of 10 RCTs by Janiaud et al. found that convalescent plasma was not associated with improved survival among COVID-19 patients (9). This latter meta-analysis was strongly affected by RECOVERY Trial data, which had a statistical weight of $90 \%$. By contrast, our pragmatic meta-analysis of RCT data showed that overall in all studies, patients transfused with convalescent plasma exhibited reduced mortality rates compared to non-transfused COVID19 patients ( 11 vs. $16 \%$ mortality; OR, $0.65 ; 95 \%$ CI, $0.43,0.98$, $P=0.04$ ) (8). For the interpretation of these results, we offer the caveat that our prior analyses excluded the trial by Agarwal et al. (30) due to the low plasma quality and was published before the release of the RECOVERY Trial data (11). Completion of several ongoing RCTs designed in accordance with the key principles of passive antibody therapy will provide more comprehensive data regarding the effect of convalescent plasma on COVID-19 patient survival.

Even among trials that did not find a survival benefit, RCTs identified positive effects of convalescent plasma on other clinical outcomes including symptomatology, oxygen requirements, and viral clearance. For example, Agarwal et al. found that a significantly greater proportion of transfused patients exhibited improved viral clearance and resolution of shortness of breath and fatigue within 1 week of randomization (30). Similarly, among patients with severe disease, Li et al. observed that transfused patients exhibited greater viral clearance and shorter times to reductions in supplemental oxygen and hospital discharge (27). Other RCTs have made similar observations (40-43, 45, 46). These additional clinical outcomes compliment positive safety data to support convalescent plasma therapy for patients hospitalized with COVID-19 $(75,76)$.

\section{Matched-Control Studies}

In contrast to RCTs, matched-control studies have generally observed a survival benefit associated with convalescent plasma therapy. Inspection of available Kaplan-Meier survival data from nine published matched-control studies showed a consistent 
pattern whereby convalescent plasma appeared to improve COVID-19 patient survival compared to non-transfused patients (Figure 1) (50, 52, 59-62, 64, 69, 70). Combining these available survival data demonstrated an $11 \%$ relative improvement in 28 day survival associated with convalescent plasma compared to control patients (82 vs. $74 \%$ ) (Figure 2C). Our recent metaanalysis that aggregated data from 20 matched-control studies supports these findings (8). These analyses indicated that patients transfused with convalescent plasma exhibited a $43 \%$ relative reduction in mortality compared to patients receiving standard treatments (21 vs. $29 \%$ mortality; OR, $0.57 ; 95 \%$ CI, 0.45 , $0.72 ; P<0.001)(8)$. The contrasting mortality benefit between matched-control studies and larger multicenter RCTs raises the possibility that locally sourced plasma administered shortly after donation may increase convalescent plasma efficacy against local variants in real time as they emerge (17). Compared to RCTs, matched-control studies are inherently predisposed to greater bias risk (77). However, several studies observed convalescent plasma efficacy using rigorous propensity matching for key variables such as age, sex, disease severity and oxygen requirements, and comorbidities $(52,64,69,70)$. For example, a large propensity-matched study by Salazar and colleagues found that transfusion of high-titer convalescent plasma within $44 \mathrm{~h}$ of hospitalization reduced mortality among patients hospitalized with COVID-19 (64).

\section{Other Clinical Evidence}

The evidence mosaic for convalescent plasma efficacy also includes data from dose-response studies and case series of vulnerable immunocompromised patient populations. On the basis that anti-SARS-CoV-2 neutralizing antibodies represent the primary mechanism for plasma efficacy, we previously examined the dose-response relationship between antibody titer level and 30-day mortality among 3,082 transfused patients via the United States Expanded Access Program for convalescent plasma (78). Transfusion of higher titer plasma was associated with lower 30-day mortality than transfusion of plasma with lower antibody titers (22 vs. $30 \%$ ) (78). Convalescent plasma conferred a survival benefit only in patients that were transfused earlier in the disease course, as this association was not observed in patients that were mechanically ventilated at the time of transfusion (78). Similarly, in the Argentine Expanded Access Program experience $(n=$ 4,719), patients transfused with convalescent plasma within 3 days of hospitalization demonstrated a $65 \%$ reduction in mortality compared to patients transfused after 7 days of hospitalization (2).

Evidence of efficacy has also emerged from studies of convalescent plasma therapy in immunocompromised patients who cannot generate an innate immune response (79). In a case series of three COVID-19 patients with X-linked agammaglobulinemia (XLA) who required supplemental oxygen, convalescent plasma transfusion was associated with rapid improvements in oxygen requirements and symptomatology in all patients (80). These data align with observations from the RECOVERY Trial subgroup analysis, which demonstrated a trend toward a reduction in mortality associated with convalescent plasma transfusion among patients that did not generate a SARS-CoV-2 antibody response (11). These observations also provide a unique "experiment of nature" for convalescent plasma, as the rapid recovery of these patients was not confounded by endogenous production of neutralizing antibodies.

\section{CLINICAL LIMITATIONS OF CONVALESCENT PLASMA THERAPY FOR COVID-19}

Challenges associated with convalescent plasma therapy likely limit clinical evidence and interpretation of the mosaic of evidence. First, the therapeutic dose per se of convalescent plasma that imposes viral neutralization and improves the recipient's clinical condition is unknown and represents a highly complicated concept. In addition, a "gold standard" serological assay to detect antibodies to SARS-CoV-2 has not been identified, and the assays in use are investigational (81). Thus, some studies likely failed to identify a clinical benefit of convalescent plasma due to low antibody levels, and between-trial differences in both antibody levels and assays may contribute to variability in outcomes between trials. Given the limitations of low antibody levels, the US Food and Drug Administration (FDA) now requires use of convalescent plasma with higher antibody levels, thereby eliminating the use of low antibody convalescent plasma (1).

Second, the precise therapeutic window of convalescent plasma that corresponds to antiviral effects is unknown but convalescent plasma likely has limited effects late in the COVID-19 disease course, for example, among patients that require mechanical ventilation. Some studies that reported no effect of convalescent plasma transfused patients that were in advanced stages of COVID-19 respiratory failure attributed to an excessive inflammatory response $(11,27,30)$. These results should not be surprising and may be interpreted as confirming the limitations of the therapeutic window of convalescent plasma rather than interpreted as "null findings" with respect to therapeutic effectiveness of plasma.

Evidence emerging from RCTs suggests that the therapeutic window of convalescent plasma coincides with that of the antiviral remdesivir, which was associated with greater patient recovery when administered prior to transition to high-flow oxygen and mechanical ventilation (82). Two other experimental COVID-19 therapies may be used to treat patients outside the therapeutic window of convalescent plasma. Prior to hospitalization, COVID-19 patients may receive monoclonal antibodies, which reduce both viral load and the time to symptom resolution in non-hospitalized individuals with COVID-19 (83, 84). Upon progression to severe COVID-19 requiring mechanical ventilation, dexamethasone represents a promising experimental therapy for stemming the inflammatory phase of COVID-19 and improving patient survival (85). 


\section{CONCLUSIONS AND FUTURE DIRECTIONS}

Currently, a mosaic of evidence from varying epistemic levels exists for convalescent plasma therapy. While most RCTs indicate that convalescent plasma confers no survival benefit when used in severely ill hospitalized COVID-19 patients, these same trials have identified clinical improvements, such as viral clearance and reduced supplemental oxygen, following plasma transfusion. In addition, although two of the largest RCTs found no effect of convalescent plasma, these trials were limited by low plasma quality (30) or a high proportion of severely ill patients concomitantly treated with corticosteroids [RECOVERY Trial (11)], which can interfere with antibody function. In contrast, worldwide matched-control studies have consistently found that convalescent plasma improves COVID-19 patient survival. Qualitative inspection of individual and aggregate Kaplan-Meier survival curves from RCTs and matched-control studies reveals a directionally consistent pattern, suggesting convalescent plasma efficacy in COVID-19 patients. Importantly, worldwide studies have consistently found that convalescent plasma therapy is as safe as standard plasma. Thus, while individual lines of evidence forming the mosaic each have foibles, such as potential bias in the observational studies and late usage of low titer plasma in some RCTs, the composite mosaic encourages the use and continued study of convalescent plasma. In this regard, there have been five major infectious disease outbreaks in 21 years, namely, SARS, MERS, Zika virus, Ebola, and COVID-19, and convalescent plasma therapy was used for all but Zika virus. Convalescent plasma epitomizes a "common sense" therapeutic, which, if implemented rapidly in future infectious disease outbreaks and in a manner that abides by the core principles of passive antibody

\section{REFERENCES}

1. US Food and Drug Administration. Clinical Memorandum for the Emergency Use Authorization of COVID-19 Convalescent Plasma (2020). Available online at: https://www.fda.gov/media/141480/download (accessed April 29, 2021).

2. González SE, Regairaz L, Salazar M, Ferrando NS, González Martínez VV, Carrera Ramos PM, et al. Timing of convalescent plasma administration and 28-day mortality for COVID-19 pneumonia. medRxiv [Preprint]. (2021). doi: 10.1101/2021.02.02.21250758

3. Duan K, Liu B, Li C, Zhang H, Yu T, Qu J, et al. Effectiveness of convalescent plasma therapy in severe COVID-19 patients. Proc Natl Acad Sci USA. (2020) 117:9490-6. doi: 10.1073/pnas.2004168117

4. Perotti C, Baldanti F, Bruno R, Del Fante C, Seminari E, Casari S, et al. Mortality reduction in 46 severe Covid-19 patients treated with hyperimmune plasma. A proof of concept single arm multicenter trial. Haematologica. (2020) 105:2834-40. doi: 10.3324/haematol.2020.261784

5. United States Food and Drug Administration. Emergency Use Authorizations for Drug and Biological Products (2021). Available online at: https://www. fda.gov/emergency-preparedness-and-response/mcm-legal-regulatory-andpolicy-framework/emergency-use-authorization\#coviddrugs (accessed April 29, 2021).

6. Chen P, Nirula A, Heller B, Gottlieb RL, Boscia J, Morris J, et al. SARS-CoV-2 neutralizing antibody LY-CoV555 in outpatients with Covid-19. N Engl J Med. (2021) 384:229-37. doi: 10.1056/NEJMoa2029849

7. Casadevall A, Grossman BJ, Henderson JP, Joyner MJ, Shoham S, Pirofski L, et al. The assessment of convalescent plasma efficacy against COVID-19. Med. (2020) 1:66-77. doi: 10.1016/j.medj.2020.11.002 therapy, can continue to serve as a stopgap therapy until more effective strategies are identified.

\section{AUTHOR CONTRIBUTIONS}

SK, JS, AC, and MJ conceived and designed the study. SK, KS, JS, PJ, RC, AC, and MJ analyzed the data and generated figures. All authors reviewed, critically revised, and approved the final version of the manuscript.

\section{FUNDING}

This study was supported by the National Heart, Lung, and Blood Institute grant (5R35HL139854, to MJ), the National Institute of Diabetes and Digestive and Kidney Diseases (5T32DK07352, to JS and CW), the Natural Sciences and Engineering Research Council of Canada (PDF-532926-2019, to SK), the National Institutes of Health (1-F32-HL154320-01 to JS), the National Institute of Allergy and Infectious Disease (R21 AI145356 and R21 AI152318, to DF; R01 AI1520789, to AC), and the National Heart, Lung, and Blood Institute (R01 HL059842, to AC).

\section{ACKNOWLEDGMENTS}

The authors express their gratitude to convalescent plasma donors.

\section{SUPPLEMENTARY MATERIAL}

The Supplementary Material for this article can be found online at: https://www.frontiersin.org/articles/10.3389/fmed. 2021.684151/full\#supplementary-material

8. Klassen SA, Senefeld JW, Johnson PW, Carter RE, Wiggins CC, Shoham S, et al. The effect of convalescent plasma therapy on COVID-19 patient mortality: systematic review and meta-analysis. Mayo Clin Proc. (2021) 96:1262-75. doi: 10.1016/j.mayocp.2021.02.008

9. Janiaud P, Axfors C, Schmitt AM, Gloy V, Ebrahimi F, Hepprich M, et al. Association of convalescent plasma treatment with clinical outcomes in patients with COVID-19: a systematic review and meta-analysis. JAMA. (2021) 325:1185-95. doi: 10.1001/jama.2021.2747

10. Chai KL, Valk SJ, Piechotta V, Kimber C, Monsef I, Doree C, et al. Convalescent plasma or hyperimmune immunoglobulin for people with COVID-19: a living systematic review. Cochrane Database Syst Rev. (2020) 7:CD013600. doi: 10.1002/14651858.CD013600.pub3

11. Horby PW, Estcourt L, Peto L, Emberson JR, Staplin N, Spata E, et al. Convalescent plasma in patients admitted to hospital with COVID-19 (RECOVERY): a randomised, controlled, open-label, platform trial. medRxiv [Preprint]. (2021). doi: 10.1101/2021.03.09.21252736

12. Casadevall A, Pirofski L, Joyner MJ. The principles of antibody therapy for infectious diseases with relevance for COVID-19. MBio. (2021) 12. doi: 10.1128/mBio.03372-20. [Epub ahead of print].

13. Casadevall A, Scharff MD. Return to the past: the case for antibodybased therapies in infectious diseases. Clin Infect Dis. (1995) 21:15061. doi: 10.1093/clinids/21.1.150

14. Bloch EM, Shoham S, Casadevall A, Sachais BS, Shaz B, Winters JL, et al. Deployment of convalescent plasma for the prevention and treatment of COVID-19. J Clin Invest. (2020) 130:2757-65. doi: 10.1172/JCI138745

15. Kaufmann SHE. Remembering Emil von Behring: From Tetanus Treatment to Antibody Cooperation With Phagocytes. mBio (2017) 8:e00117-17. 
16. Walensky RP, Walke HT, Fauci AS. SARS-CoV-2 variants of concern in the United States-challenges and opportunities. JAMA. (2021) 325:10378. doi: 10.1001/jama.2021.2294

17. Ratcliff J, Nguyen D, Fish M, Rhynne J, Jennings A, Williams S, et al. Virological and serological characterization of critically ill patient with COVID-19 in the UK: a special focus on variant detection. medRxiv [Preprint]. (2021). doi: 10.1101/2021.02.24.21251989

18. Casadevall A, Henderson J, Joyner M, Pirofski L. SARS-Cov2 variants and convalescent plasma: reality, fallacies, and opportunities. J Clin Invest. (2021) 131:e148832. doi: 10.1172/JCI148832

19. Kunze KL, Johnson PW, van Helmond N, Senefeld JW, Petersen MM, Klassen SA, et al. Mortality in individuals treated with COVID-19 convalescent plasma varies with the geographic provenance of donors. medRxiv [Preprint]. (2021). doi: 10.1101/2021.03.19.21253975

20. Casadevall A, Pirofski L. The convalescent sera option for containing COVID19. J Clin Invest. (2020) 130:1545-8. doi: 10.1172/JCI138003

21. Flexner $\mathrm{S}$. The results of the serum treatment in thirteen hundred cases of epidemic meningitis. J Exp Med. (1913) 17:553-76. doi: 10.1084/jem.17.5.553

22. CECIL RL. Effects of very early serum treatment in pneumococcus type I pneumonia. J Am Med Assoc. (1937) 108:68992. doi: $10.1001 /$ jama.1937.02780090001001

23. Luke TC, Kilbane EM, Jackson JL, Hoffman SL. Meta-analysis: convalescent blood products for Spanish influenza pneumonia: a future H5N1 treatment? Ann Intern Med. (2006) 145:599609. doi: 10.7326/0003-4819-145-8-200610170-00139

24. Cheng Y, Wong R, Soo YOY, Wong WS, Lee CK, Ng MHL, et al. Use of convalescent plasma therapy in SARS patients in Hong Kong. Eur J Clin Microbiol Infect Dis. (2005) 24:44-6. doi: 10.1007/s10096-004-1271-9

25. Ko J-H, Seok H, Cho SY, Ha YE, Baek JY, Kim SH, et al. Challenges of convalescent plasma infusion therapy in Middle East respiratory coronavirus infection: a single centre experience. Antivir Ther. (2018) 23:61722. doi: 10.3851/IMP3243

26. Yeh K-M, Chiueh T-S, Siu LK, Lin J-C, Chan PKS, Peng M-Y, et al. Experience of using convalescent plasma for severe acute respiratory syndrome among healthcare workers in a Taiwan hospital. J Antimicrob Chemother. (2005) 56:919-22. doi: 10.1093/jac/dki346

27. Li L, Zhang W, Hu Y, Tong X, Zheng S, Yang J, et al. Effect of convalescent plasma therapy on time to clinical improvement in patients with severe and life-threatening COVID-19: a randomized clinical trial. JAMA. (2020) 324:460-70. doi: 10.1001/jama.2020.10044

28. Robbiani DF, Gaebler C, Muecksch F, Lorenzi JCC, Wang Z, Cho A. Convergent antibody responses to SARS-CoV-2 in convalescent individuals. Nature. (2020) 584:437-42. doi: 10.1038/s41586-020-2456-9

29. Natarajan H, Crowley AR, Butler SE, Xu S, Weiner JA, Bloch EM, et al. SARS-CoV-2 antibody signatures robustly predict diverse antiviral functions relevant for convalescent plasma therapy. medRxiv [Preprint]. (2020). doi: 10.1101/2020.09.16.20196154

30. Agarwal A, Mukherjee A, Kumar G, Chatterjee P, Bhatnagar T, Malhotra P. Convalescent plasma in the management of moderate covid-19 in adults in India: open label phase II multicentre randomised controlled trial (PLACID Trial). BMJ. (2020) 371:m3939. doi: 10.1136/bmj.m3939

31. Pirofski L, Casadevall A. Pathogenesis of COVID-19 from the perspective of the damage-response framework. MBio. (2020) 11. doi: $10.1128 / \mathrm{mBio} .01175-20$. [Epub ahead of print].

32. Wang Y, Zhang L, Sang L, Ye F, Ruan S, Zhong B, et al. Kinetics of viral load and antibody response in relation to COVID-19 severity. J Clin Invest. (2020) 130:5235-44. doi: 10.1172/JCI138759

33. Casadevall A, Pirofski L. In fatal COVID-19, the immune response can control the virus but kill the patient. Proc Natl Acad Sci. (2020) 117:3000911. doi: $10.1073 /$ pnas.2021128117

34. Wu M, Chen Y, Xia H, Wang C, Tan CY, Cai X, et al. Transcriptional and proteomic insights into the host response in fatal COVID-19 cases. Proc Natl Acad Sci. (2020) 117:28336-43. doi: 10.1073/pnas.2018030117

35. Bandopadhyay P, Rozario RD, Lahiri A, Sarif J, Ray Y, Paul SR, et al. Nature and dimensions of the systemic hyper-inflammation and its attenuation by convalescent plasma in severe COVID-19. J Infect Dis. (2021). doi: 10.1101/2020.09.21.20199109. [Epub ahead of print].
36. Tremblay D, Seah C, Schneider T, Bhalla S, Feld J, Naymagon L, et al. Convalescent plasma for the treatment of severe COVID-19 infection in cancer patients. Cancer Med. (2020) 9:8571-8. doi: 10.1002/cam4.3457

37. Imai M, Iwatsuki-Horimoto K, Hatta M, Loeber S, Halfmann PJ, Nakajima $\mathrm{N}$, et al. Syrian hamsters as a small animal model for SARS-CoV-2 infection and countermeasure development. Proc Natl Acad Sci USA. (2020) 117:1658795. doi: 10.1073/pnas.2009799117

38. Cross RW, Prasad AN, Borisevich V, Woolsey C, Agans KN, Deer DJ, et al. Use of convalescent serum reduces severity of COVID-19 in nonhuman primates. Cell Rep. (2021) 34:108837. doi: 10.1016/j.celrep.2021.108837

39. Sun J, Zhuang Z, Zheng J, Li K, Wong RLY, Liu D, et al. Generation of a broadly useful model for COVID-19 pathogenesis, vaccination, and treatment. Cell. (2020) 182:734-43.e5. doi: 10.1016/j.cell.2020.06.010

40. Bajpai M, Maheshwari A, Chabra K, Kale P, Gupta A, Gupta E, et al. Efficacy of convalescent plasma therapy compared to fresh frozen plasma in severely ill COVID-19 patients: a pilot randomized controlled trial. medRxiv [Preprint]. (2020). doi: 10.1101/2020.10.25.20219337

41. Avendano-Sola C, Ramos-Martinez A, Munez-Rubio E, Ruiz-Antoran B, de Molina RM, Torres F, et al. Convalescent plasma for COVID19: a multicenter, randomized clinical trial. medRxiv [Preprint]. (2020). doi: 10.1101/2020.08.26.20182444

42. Rasheed AM, Fatak DF, Hashim HA, Maulood MF, Kabah KK, Abdulamir AS, et al. The therapeutic potential of convalescent plasma therapy on treating critically-ill COVID-19 patients residing in respiratory care units in hospitals in Baghdad, Iraq. Le Infez Med. (2020) 28:35766. doi: 10.1101/2020.06.24.20121905

43. Gharbharan A, Jordans CCE, GeurtsvanKessel C, den Hollander JG, Karim F, Mollema FPN, et al. Convalescent plasma for COVID-19. A randomized clinical trial. medRxiv [Preprint]. (2020). doi: 10.1101/2020.07.01.201 39857

44. AlQahtani M, Abdulrahman A, AlMadani A, AlAli SY, Al Zamrooni AM, Hejab A, et al. Randomized controlled trial of convalescent plasma therapy against standard therapy in patients with severe COVID-19 disease. medRxiv [Preprint]. (2020). doi: 10.1101/2020.11.02.20224303

45. Libster R, Pérez Marc G, Wappner D, Coviello S, Bianchi A, Braem V, et al. Early high-titer plasma therapy to prevent severe Covid-19 in older adults. $N$ Engl J Med. (2021) 384:610-8. doi: 10.1056/NEJMoa2033700

46. Ray Y, Paul SR, Bandopadhyay P, D’Rozario R, Sarif J, Lahiri A, et al. Clinical and immunological benefits of convalescent plasma therapy in severe COVID19: insights from a single center open label randomised control trial. medRxiv [Preprint]. (2020). doi: 10.1101/2020.11.25.20237883

47. Simonovich VA, Burgos Pratx LD, Scibona P, Beruto M V, Vallone $\mathrm{MG}$, Vázquez $\mathrm{C}$, et al. A randomized trial of convalescent plasma in Covid-19 severe pneumonia. N Engl J Med. (2020) 384:619-29. doi: 10.1056/NEJMoa2031304

48. Xia X, Li K, Wu L, Wang Z, Zhu M, Huang B, et al. Improved clinical symptoms and mortality among patients with severe or critical COVID-19 after convalescent plasma transfusion. Blood. (2020) 136:7559. doi: 10.1182/blood.2020007079

49. Abolghasemi H, Eshghi P, Cheraghali AM, Imani Fooladi AA, Bolouki Moghaddam F, Imanizadeh S, et al. Clinical efficacy of convalescent plasma for treatment of COVID-19 infections: results of a multicenter clinical study. Transfus Apher Sci. (2020) 59:102875. doi: 10.1016/j.transci.2020.102875

50. AlShehry N, Zaidi SZA, AlAskar A, Al Odayani A, Alotaibi JM, AlSagheir A, et al. Safety and efficacy of convalescent plasma for severe COVID-19: interim report of a multicenter phase II study from Saudi Arabia. Saudi J Med Med Sci. (2021) 9:16. doi: 10.4103/sjmms.sjmms_731_20

51. Budhiraja S, Dewan A, Aggarwal R, Singh O, Juneja D, Pathak S, et al. Effectiveness of Convalescent Plasma Therapy in Indian Patients with COVID19. doi: $10.2139 /$ ssrn. 3726179

52. Yoon H ah, Bartash R, Gendlina I, Rivera J, Nakouzi A, Bortz III RH, et al. Treatment of severe COVID-19 with convalescent plasma in Bronx, NYC. JCI Insight. (2021) 6:e142270. doi: 10.1172/jci.insight.142270

53. Rogers R, Shehadeh F, Mylona E, Rich J, Neill M, Touzard-Romo F, et al. Convalescent plasma for patients with severe COVID-19: a matched cohort study. medRxiv [Preprint]. (2020). doi: 10.1101/2020.08.18.201 77402 
54. Altuntas F, Ata N, Yigenoglu TN, Basci S, Dal MS, Korkmaz S, et al. Convalescent plasma therapy in patients with COVID-19. Transfus Apher Sci. (2020) 60:102955. doi: 10.1016/j.transci.2020.102955

55. Klapholz M, Pentakota SR, Zertuche J-P, McKenna M, Roque W, Forsberg $\mathrm{M}$, et al. Matched cohort study of convalescent COVID-19 plasma (CCP) treatment in severely or life threateningly Ill COVID-19 patients. Open Forum Infect Dis. (2021) 8:ofab001. doi: 10.1093/ofid/ofab001

56. Klein MN, Wang EW, Zimand P, Beauchamp H, Donis C, Ward $\mathrm{MD}$, et al. Kinetics of SARS-CoV-2 antibody responses pre-and postCOVID-19 convalescent plasma transfusion in patients with severe respiratory failure: an observational case-control study. medRxiv [Preprint]. (2020). doi: 10.1101/2020.12.10.20247007

57. Moniuszko-Malinowska A, Czupryna P, Zarebska-Michaluk D, Tomasiewicz $\mathrm{K}$, Pancewicz S, Rorat M, et al. Convalescent plasma transfusion for the treatment of COVID-19-Experience from Poland: a multicenter study. J Clin Med. (2021) 10:28. doi: 10.3390/jcm10010028

58. Omrani AS, Zaqout A, Baiou A, Daghfal J, Elkum N, Alattar RA, et al. Convalescent plasma for the treatment of patients with severe coronavirus disease 2019: a preliminary report. J Med Virol. (2020) 93:167886. doi: $10.1002 /$ jmv. 26537

59. Kurtz P, Righy C, Gadelha M, Bozza FA, Bozza PT, Gonçalves B, et al. Effect of convalescent plasma in critically ill patients with COVID-19: an observational study. Front Med. (2021) 8:630982. doi: 10.3389/fmed.2021.630982

60. Shenoy AG, Hettinger AZ, Fernandez SJ, Blumenthal J, Baez V. Early mortality benefit with COVID-19 convalescent plasma: a matched control study. $\mathrm{Br} \mathrm{J}$ Haematol. (2021) 192:706-13. doi: 10.1111/bjh.17272

61. Sturek JM, Thomas TA, Gorham JD, Sheppard CA, Raymond AE, De Guex KP, et al. Convalescent plasma for preventing critical illness in COVID-19: a phase 2 trial and immune profile. medRxiv [Preprint]. (2021). doi: 10.1101/2021.02.16.21251849

62. Thompson MA, Henderson JP, Shah PK, Rubinstein SM, Joyner MJ, Choueiri TK, et al. Convalescent plasma and improved survival in patients with hematologic malignancies and COVID-19. medRxiv [Preprint]. (2021). doi: 10.1101/2021.02.05.21250953

63. Hegerova L, Gooley TA, Sweerus KA, Maree C, Bailey N, Bailey M, et al. Use of convalescent plasma in hospitalized patients with COVID-19: case series. Blood. (2020) 136:759-62. doi: 10.1182/blood.2020006964

64. Salazar E, Christensen PA, Graviss EA, Nguyen DT, Castillo B, Chen J, et al. Significantly decreased mortality in a large cohort of coronavirus disease 2019 (COVID-19) patients transfused early with convalescent plasma containing high-titer anti-severe acute respiratory syndrome coronavirus 2 (SARS-CoV-2) spike protein IgG. Am J Pathol. (2021) 191:90107. doi: 10.1016/j.ajpath.2020.10.008

65. Alsharidah S, Ayed M, Ameen RM, Alhuraish F, Rouheldeen NA, Alshammari FR, et al. COVID-19 convalescent plasma treatment of moderate and severe cases of SARS-CoV-2 infection: a multicenter interventional study. Int J Infect Dis. (2020) 103:439-46. doi: 10.1016/j.ijid.2020.11.198

66. Zeng QL, Yu ZJ, Gou JJ, Li GM, Ma SH, Zhang GF, et al. Effect of convalescent plasma therapy on viral shedding and survival in patients with coronavirus disease 2019. J Infect Dis. (2020) 222:38-43. doi: 10.1093/infdis/jiaa228

67. Donato M, Park S, Baker M, Korngold R, Morawski A, Geng X, et al. Clinical and laboratory evaluation of patients with SARS-CoV-2 pneumonia treated with high-titer convalescent plasma: a prospective study. medRxiv [Preprint]. (2020). doi: 10.1101/2020.07.20.20156398

68. Salazar MR, Gonzalez SE, Regairaz L, Ferrando NS, Gonzalez V, Carrera PM, et al. Effect of convalescent plasma on mortality in patients with COVID19 pneumonia. medRxiv [Preprint]. (2020). doi: 10.1101/2020.10.08.202 02606

69. Liu STH, Lin HM, Baine I, Wajnberg A, Gumprecht JP, Rahman F, et al. Convalescent plasma treatment of severe COVID-19: a propensity score-matched control study. Nat Med. (2020) 26:1708-13. doi: 10.1038/s41591-020-1088-9

70. Tworek A, Jaroń K, Uszyńska-Kałuza B, Rydzewski A, Gil R, Deptała A, et al. Convalescent plasma treatment is associated with lower mortality and better outcomes in high risk COVID-19 patients-propensity score matched casecontrol study. Int J Infect Dis. (2021) 105:209-15. doi: 10.1016/j.ijid.2021. 02.054
71. O’Donnell M, Grinsztejn B, Cummings M, Justman J, Lamb M, Eckhardt C, et al. A randomized, double-blind, controlled trial of convalescent plasma in adults with severe COVID-19. medRxiv [Preprint]. (2021). doi: 10.1101/2021.03.12.21253373

72. Greenwood M. The Natural Duration of Cancer (Report on Public Health and Medical Subjects no 33). London Station Off. (1926).

73. Guyot P, Ades AE, Ouwens MJNM, Welton NJ. Enhanced secondary analysis of survival data: reconstructing the data from published Kaplan-Meier survival curves. BMC Med Res Methodol. (2012) 12:1-13. doi: 10.1186/1471-2288-12-9

74. Bhandari R, Khanna G, Kuhad A. Pharmacological insight into potential therapeutic agents for the deadly Covid-19 pandemic. Eur J Pharmacol. (2021) 890:173643. doi: 10.1016/j.ejphar.2020.173643

75. Joyner M, Wright RS, Fairweather D, Senefeld J, Bruno K, Klassen S, et al. Early safety indicators of COVID-19 convalescent plasma in 5,000 patients. $J$ Clin Invest. (2020) 30:4791-7. doi: 10.1172/JCI140200

76. Joyner MJ, Bruno KA, Klassen SA, Kunze KL, Johnson PW, Lesser ER, et al. Safety update: COVID-19 convalescent plasma in 20,000 hospitalized patients. Mayo Clin Proc. (2020) 95:1888-97. doi: 10.1016/j.mayocp.2020.06.028

77. Califf RM, Hernandez AF, Landray M. Weighing the benefits and risks of proliferating observational treatment assessments: observational cacophony, randomized harmony. JAMA. (2020) 324:625-6. doi: 10.1001/jama.2020.13319

78. Joyner MJ, Carter RE, Senefeld JW, Klassen SA, Mills JR, Johnson PW, et al. Convalescent plasma antibody levels and the risk of death from covid-19. N Engl J Med. (2021) 384:1015-27. doi: 10.1056/NEJMoa2031893

79. Senefeld J, Klassen SA, Ford SK, Wiggins CC, Bostrom BC, Thompson MA, et al. Therapeutic use of convalescent plasma in COVID-19 patients with immunodeficiency: a systematic review. medRxiv [Preprint]. (2020). doi: 10.1101/2020.11.08.20224790

80. Jin H, Reed JC, Liu STH, Ho H en, Lopes JP, Ramsey NB, et al. Three patients with X-linked agammaglobulinemia hospitalized for COVID-19 improved with convalescent plasma. J Allergy Clin Immunol Pract. (2020) 8:35946.e3. doi: 10.1016/j.jaip.2020.08.059

81. US Food and Drug Administration (FDA). Emergency Use Authorization (EUA) for the Emergency Use of COVID-19 Convalescent Plasma for the Treatment of Hospitalized Patients With Coronavirus Disease 2019 (COVID19) (2021). Available online at: https://www.fda.gov/media/141477/download (accessed April 29, 2021).

82. Benfield T, Fätkenheuer G, Kortepeter MG, Atmar RL, Creech CB, Lundgren J, et al. Remdesivir for the treatment of COVID-19-Final report. N Engl J Med. (2020) 383:1813-26. doi: 10.1056/NEJMoa2007764

83. Weinreich DM, Sivapalasingam S, Norton T, Ali S, Gao H, Bhore R, et al. REGN-COV2, a neutralizing antibody cocktail, in outpatients with Covid-19. N Engl J Med. (2021) 384:238-51. doi: 10.1056/NEJMoa2035002

84. Regeneron. Phase 3 Trial Shows REGEN-COV ${ }^{T M}$ (casirivimab With Imdevimab) Antibody Cocktail Reduced Hospitalization or Death by $70 \%$ in Non-hospitalized COVID-19 Patients (2021). Available online at: https:// investor.regeneron.com/news-releases/news-release-details/phase-3-trialshows-regen-covtm-casirivimab-imdevimab-antibody (accessed April 29, 2021).

85. RECOVERY Collaborative Group. Dexamethasone in hospitalized patients with Covid-19-preliminary report. N Engl J Med. (2020) 84:693704. doi: 10.1056/NEJMoa2021436

Conflict of Interest: The authors declare that the research was conducted in the absence of any commercial or financial relationships that could be construed as a potential conflict of interest.

Copyright () 2021 Klassen, Senefeld, Senese, Johnson, Wiggins, Baker, van Helmond, Bruno, Pirofski, Shoham, Grossman, Henderson, Wright, Fairweather, Paneth, Carter, Casadevall and Joyner. This is an open-access article distributed under the terms of the Creative Commons Attribution License (CC BY). The use, distribution or reproduction in other forums is permitted, provided the original author(s) and the copyright owner(s) are credited and that the original publication in this journal is cited, in accordance with accepted academic practice. No use, distribution or reproduction is permitted which does not comply with these terms. 